\title{
Avoidable deficiencies in the delivery of health care to children with meningococcal disease
}

\author{
Simon Nadel, Joseph Britto, Robert Booy, Ian Maconochie, Parviz Habibi, Michael Levin
}

\begin{abstract}
Objectives-It is apparent that delays and inadequate or inappropriate management occur frequently and may contribute to the continued high mortality seen in meningococcal disease. An attempt has been made to define the major sources of delay or inappropriate treatment.

Methods-A prospective, descriptive study of children with meningococcal disease referred to a tertiary centre paediatric intensive care and infectious disease unit. Definitions of optimal care were established at three stages: parental; general practitioner (GP)/accident and emergency (A\&E) department; and hospital. Duration of symptoms and management were recorded from direct questioning of parents and carers, and from hospital records.
\end{abstract}

Results-54 consecutive children with meningococcal disease were recruited to the study. Delayed parental recognition occurred in 16 children. GPs correctly diagnosed 19 of 35 children. Delay of 2.5-21 hours occurred in those who were incorrectly diagnosed. Two of 15 children who presented to the A\&E department with specific features were incorrectly diagnosed. Hospital treatment was suboptimal in $71 \%$. Shock was not recognised or treated in $50 \%, 20 \%$ of children had unnecessary lumbar punctures. Time from illness onset to treatment was longer in fatal disease (median 18.3, range 8-24 hours), compared with survivors (median 12, range 2-48 hours; $p<0.01$, MannWhitney U test).

Conclusion-Suboptimal treatment in meningococcal disease is due to failure of parents, GPs, and hospital doctors to recognise specific features of the illness. Improvement in outcome could be achieved by public education and better training of clinicians in recognition, resuscitation, and stabilisation of seriously ill children.

(F Accid Emerg Med 1998;15:298-303)

Keywords: meningococcal disease; avoidable deficiences; health care delivery

Meningococcal disease remains an important cause of childhood morbidity and mortality in the UK. Each year there are over 2000 reported cases, most of which occur in young children. ${ }^{1}$ Despite the availability of potent antibiotics and improvements in paediatric critical care medicine, the overall mortality for patients with meningococcal infection remains at $10 \%$, increasing to $50 \%$ for those who present with meningococcal septic shock. ${ }^{2}$

Meningococcal infection is characteristically fulminant and may lead to death in a matter of hours. Therefore any delay in initiation of appropriate treatment is likely to have significant effects on outcome.

There are a number of stages in the interaction between a sick child and the health care system, at which management decisions may critically affect the speed with which medical treatment is initiated.

Delays in the administration of appropriate medical care to seriously ill children may be experienced:

(1) At home, if parents fail to appreciate the signs of serious illness in their child, or the significance of the specific signs of meningococcal infection, such as a petechial rash.

(2) Once a parent has recognised that their child requires medical attention, difficulties may be experienced in consulting with their doctor. A general practitioner (GP) may be unable to appreciate the urgency of the situation because the parents may be unable to convey the information adequately over the telephone. Once the doctor has seen the child, the signs of serious illness may not be recognised, due either to their doctor's lack of familiarity with the condition, or to inexperience of junior accident and emergency (A\&E) doctors. Even if meningococcal disease is recognised, further delays in the administration of antibiotics or in transporting the child to hospital may occur.

(3) Once the child is admitted to hospital, clinicians may be unfamiliar with the signs of shock or raised intracranial pressure (ICP) in children, and inappropriate management of these life threatening complications may occur. Furthermore, delays may be experienced in gaining access to an appropriate paediatric intensive care unit (PICU).

As a tertiary referral centre for paediatric infectious diseases and paediatric intensive care, with a major research interest in meningococcal disease, we have participated in the care of many children with meningococcal infection. We have noted that unnecessary delays in administration of appropriate medical care, or inappropriate or inadequate management, both in the community and in hospital, occur frequently, and may contribute to the continued high mortality from meningococcal disease.

\section{Aim}

In order to evaluate the frequency of delays and suboptimal management in children with meningococcal disease, we have carried out a 
prospective study of the first 54 children admitted to a tertiary centre paediatric unit with this diagnosis between January 1992 and January 1994.

\section{Patients and methods}

Meningococcal disease was diagnosed in children with a typical clinical presentation, that is, a petechial or purpuric rash and fever. The diagnosis was confirmed by isolation of Neisseria meningitidis from blood, cerebrospinal fluid (CSF), or nasopharyngeal culture; or in culture negative cases, by detection of Gram negative diplococci in CSF, or detection of meningococcal capsular polysaccharide in CSF or blood by latex agglutination.

Patients with typical clinical features of meningococcal disease, but where microbiological investigations were negative were included if no alternative aetiology was identified after full microbiological investigation.

Patients were diagnosed with meningococcal septicaemia if they had fever and a petechial or purpuric rash and features of systemic infection (tachycardia, tachypnoea, hypothermia, or hyperthermia). Shock was diagnosed by the presence of hypotension (blood pressure $<3$ rd centile for age), or in the absence of hypotension, two or more features of inadequate tissue perfusion, including: base deficit $>8 \mathrm{mmol} / 1$; capillary refill time $>5 \mathrm{sec}$; core-peripheral temperature gradient $>3^{\circ} \mathrm{C}$; oliguria $<0.5$ $\mathrm{ml} / \mathrm{kg} /$ hour; hypoxia (oxygen saturation $<95 \%$ in air), or deteriorating neurological status. ${ }^{3}$

Patients were diagnosed with meningococcal meningitis if they had fever and a petechial or purpuric rash with meningism (stiff neck, photophobia, positive Kernig's sign, depressed consciousness), without the features of shock. As we do not routinely perform lumbar puncture in the acute phase of illness in a child with a petechial or purpuric rash, particularly if there is any evidence of circulatory insufficiency, we would presume the presence of meningitis in the presence of the above clinical findings.

In those children who did undergo lumbar puncture, meningococcal meningitis was confirmed by the presence of CSF findings consistent with bacterial meningitis and bacteriological confirmation, or in the absence of positive culture or rapid antigen results, presumed meningococcal infection as defined above.

Patients with clinical or laboratory evidence of both meningitis and septicaemia were included in the septicaemia group.

Information regarding the child's condition before hospitalisation was obtained by direct questioning of parents and carers, and where possible from GP referral letters. Details of the child's condition and management after admission to hospital was gathered prospectively from the medical and nursing records of the first admitting hospital, from our own records for patients who were direct admissions to our hospital and for tertiary referrals.
DEFINITIONS OF APPROPRIATE MEDICAL CARE Recognition and management of children with meningococcal disease was evaluated at the following three stages of health care delivery.

\section{(1) Recognition by parents}

The following features were defined as criteria that should have enabled parents to recognise the need to seek medical advice: fever with petechial or purpuric rash; or fever with at least two of the following signs or symptoms of serious illness: cyanosis; rigors; convulsions; confusion, delirium or altered mental state; severe headache; severe muscle or joint pain; severe weakness (that is inability to walk or stand); persistent vomiting (three or more occasions within 12 hours); and cold extremities.

The duration of time from appearance of these symptoms, to when parents first sought medical advice from their GP or A\&E department, was determined by direct questioning of the parent or guardian as soon as possible after hospital admission. Where possible both parents were questioned, together with other family members or friends involved in the child's care. Accuracy of recollection of the child's symptoms was confirmed by other members of the family, and information derived from GP letters or $A \& E$ records and by examining medical and nursing records from both the referral hospital and the PICU.

\section{(2) Recognition and treatment by GPs and $A \mathcal{E} E$} departments

Features of illness that should have been recognised by GPs and A\&E officers as requiring immediate treatment and referral to hospital or the paediatric service were the same as those defined above for parents. Appropriate initial treatment for meningococcal disease by GPs and A\&E officers was defined using the Department of Health guidelines. ${ }^{4}$ The first physician to see the patient should administer parenteral penicillin if the child has a petechial or purpuric rash with fever, or clinical features of meningitis. The child should then be immediately referred to hospital. In the absence of a petechial or purpuric rash, but in the presence of the features of serious illness as defined for parents, the child should have been immediately referred to hospital.

The duration of time between medical consultation and administration of appropriate initial treatment was noted.

\section{(3) Recognition and management in hospital} Clinical criteria for diagnosis of meningococcal disease by paediatricians or other hospital based physicians were fever with petechial or purpuric rash, or in the absence of rash, clinical suspicion of sepsis or meningitis. Additional features which should have enabled clinicians to recognise impending or actual shock or raised ICP were: (a) shock: see criteria above and (b) raised ICP: Glasgow coma scale <8; cardiovascular instability (hypertension and bradycardia); abnormal respiratory pattern or respiratory depression; asymmetrical, dilated, or sluggish papillary responses; cranial nerve palsy; abnormal posture; and papilloedema. ${ }^{5}$ 
Appropriate initial management of shock in the admitting hospital was defined as administration of supplemental oxygen treatment or early, elective intubation and mechanical ventilation if indicated (that is by impending or actual respiratory, cardiovascular, or neurological failure); volume replacement with crystalloid or colloidal solutions to correct hypovolaemia $(20 \mathrm{ml} / \mathrm{kg}$ or more, given rapidly); avoidance of lumbar puncture, which is recognised to be contraindicated in patients with unstable respiratory or cardiovascular parameters. $^{5}$

Appropriate initial management of raised ICP included avoidance of lumbar puncture; elective tracheal intubation and hyperventilation; fluid restriction; and administration of diuretic treatment. ${ }^{6}$

\section{Results}

Fifty four children were admitted to the PICU or paediatric infectious disease unit between January 1992 and January 1994. There were 31 boys and 23 girls, age range 1 week to 15.7 years (median 2.95 years). Fifty one were referred from other hospitals and three were admitted directly.

Forty two children had meningococcal septicaemia and 12 had meningococcal meningitis. Fifteen of those with septicaemia also had clinical or laboratory evidence of meningitis.

In 37 children, meningococcal disease was confirmed microbiologically. Ten of the 17 children with negative microbiological results were treated with parenteral antibiotics before microbiological investigation. Of those with proved infection, 18 had group B and 19 had group C $N$ meningitidis.

Time from illness onset to initiation of parenteral antibiotic treatment ranged from 2 to 96 hours (median 18.7 hours). Children with meningitis had longer duration of symptoms before presentation (median 24, range 12-96 hours) compared with those with septicaemia (median 14, range 2-48 hours; $p<$ 0.05, Mann-Whitney U test).

Thirteen children died (24\%), all with meningococcal septicaemia. The mortality rate for this group was therefore $31 \%(13 / 42)$. No child who had meningococcal meningitis without features of septicaemia died.

\section{DELAYED OR INAPPROPRIATE TREATMENT}

Using the criteria defined above to indicate the time when parents should have sought medical attention, delayed presentation to primary medical services occurred in 16 children $(30 \%)$. In six cases, parents hesitated to call their GP or take the child to A\&E during the night or at weekends. They preferred to wait, relying on repeated doses of antipyretics or tepid sponging and seek medical advice at a more "socially acceptable" time. Eight sets of parents did not appreciate the significance of a non-blanching rash, thinking the child had developed "insect bites" or "measles".

In all instances of parental delay, parents were unaware of the signs of serious illness in their child, such as rigors, persistent vomiting, or alterations in the level of consciousness. In four cases, worried parents were inappropriately reassured by advice given over the telephone by GPs or A\&E staff without the child being assessed.

Thirty seven children initially presented to their GP: 35 had signs of meningococcal disease; $11(31 \%)$ of these were appropriately treated with parenteral penicillin and promptly referred to hospital.

Delayed recognition or suboptimal treatment of meningococcal disease occurred in 24 children (65\%): eight with typical clinical features of meningococcal disease were referred to hospital without receiving parenteral penicillin from the GP; 16 were not recognised as having meningococcal disease despite the presence of fever, a petechial or purpuric rash, and clinical features of serious illness as defined above. These children were neither treated with antibiotics nor referred to hospital. Their eventual presentation and initiation of treatment was delayed by between 2 and 21 hours (median 8.5 hours). Nine children had repeated visits to their GP and seven others were subsequently taken to the $A \& E$ department without further attempts to see their GP.

Two children who initially consulted their GP had non-specific illness. When they subsequently developed the typical features of meningococcal disease they were appropriately managed.

Seventeen children presented directly to the A\&E department: 15 had typical clinical features of meningococcal disease on presentation. In two of these an incorrect alternative diagnosis was made (idiopathic thrombocytopenic purpura in one case and rubella in the other), and therefore antibiotic treatment was withheld. In one other case, although meningococcal disease was correctly diagnosed, initiation of antibiotic treatment was delayed for four hours. These three children had between 2.5 to 12 hours delay before administration of appropriate treatment.

Twelve $(71 \%)$ children had appropriate treatment in the A\&E department. Two children presented to $A \& E$ with non-specific features of illness.

All children presenting initially to the $A \& E$ department were first assessed by a senior house officer in A\&E. The two cases where an incorrrect diagnosis was initially made were reviewed by a paediatric registrar. The diagnosis was corrected when the child's clinical condition deteriorated.

All 54 children were eventually admitted to hospital under the care of paediatricians. Suboptimal management occurred in 29 children (54\%). All of these had meningococcal septicaemia. Failure to recognise shock in children with meningococcal septicaemia occurred in 19 of 38 children, resulting in inadequate resuscitation. Lumbar puncture was performed in 10 children with clinical features of shock, and in one child with features of raised ICP. Of these, nine suffered cardiovascular or neurological deterioration after this procedure, including increase in inotrope requirement, 


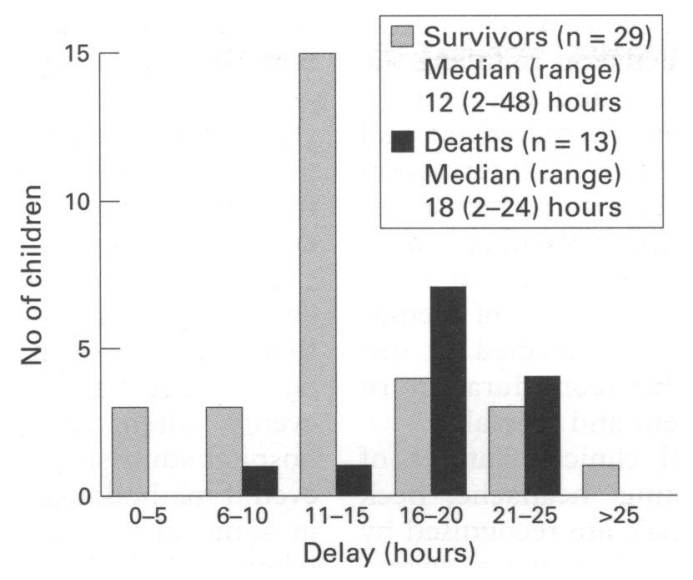

Figure 1 Time from illness onset to initiation of treatment in children with meningococcal septicaemia.

worsening coma, and one child suffered a cardiorespiratory arrest and subsequent brain death.

Only 13 of 42 children (29\%) with meningococcal septicaemia received optimal treatment after hospital admission. There was one death among this group of children.

Among children with meningococcal septicaemia, the duration of time from illness onset until initiation of appropriate treatment was longer in those who died (median 18.3, range 8-24 hours), compared with survivors (median 12 , range 2-48 hours; $p<0.01$, MannWhitney U test; fig 1).

\section{Discussion}

Any study that is undertaken to define inadequacies in health care delivery to children with meningococcal disease inevitably risks appearing judgmental or critical of the individuals involved in their care. The purpose of this study was not to apportion blame, but to attempt to systematically evaluate the frequency of delayed diagnosis or suboptimal management in children with meningococcal disease. This is a necessary prerequisite before consideration of whether improvements in both public and medical education are required to achieve an improvement in outcome.

We have shown that there are significant deficiencies of health care delivery to children with meningococcal disease. These deficiencies consist of delayed recognition of meningococcal disease by parents, GPs and hospital doctors, together with suboptimal hospital management. These factors appear to be present at all stages of illness progression.

One of the major difficulties encountered during this study was the lack of reliable indicators of the child's condition before arrival at hospital. We attempted to be objective by defining the signs and symptoms that should have led parents to consult medical attention, as those commonly accepted as indicating serious disease in children. ${ }^{7}$ Although it was sometimes difficult to obtain an accurate description of clinical features from parents, who are often extremely distressed, we found that most parents had an acute recollection of their actions and the child's condition at each stage of the illness. There was general agree- ment between events described by parents, or friends and relatives who may have accompanied them.

The reasons for parental delay in seeking medical attention are manifold. In most cases we did not delve deeply into the reasons why the doctor was not called or the child was not taken to the $A \& E$ department, as it was very difficult to suggest, at this most difficult time, that the parents should have sought medical attention earlier. However, delay in seeking medical attention due to failure of parents to appreciate the severity of illness occurred in 16 children. We were struck by how tolerant parents could be of even severe symptoms in their child. In addition, if medical advice had been sought, either in person or over the telephone, and was reassuring that the child was suffering from a "trivial infection", parents would hesitate to question the doctor's diagnosis and possibly delay seeking further advice when the child's condition subsequently deteriorated.

One of the major difficulties in the early recognition of meningococcal disease is that early symptoms are often indistinguishable from those of other common childhood febrile illnesses such as influenza or viral exanthems. ${ }^{8}$ Any public education campaign that aims to encourage parents to seek medical attention early in the course of suspected meningococcal infection inevitably risks burdening GPs and $\mathrm{A} \& \mathrm{E}$ departments with large numbers of worried parents of children with trivial illnesses. However, from our questioning of parents it seems likely that carefully worded, specific advice on the recognition of serious illness in children, and on the recognition of the rash, may help to reduce the duration of time before children with meningococcal infection received medical attention. Such information is readily available from the meningitis charities, the Meningitis Research Foundation and the National Meningitis Trust. Simple explanations as to the significance of a non-blanching rash (the "tumbler test") and the need for repeated parental review and action in the presence of continuing fever, vomiting, or severe myalgia, which should prompt further consultation with the GP or A\&E department, have been shown to improve the time to receiving medical attention (personal communication, Meningitis Research Foundation). Also, clear recommendations from the GP or $A \& E$ officer about what symptoms or signs to watch for, and what to do in the event of any deterioration in the child's condition, may prevent further delays, even if initial medical advice was reassuring. This would then empower the parents to take further action, even if it was contrary to the initial medical opinion. It may be reasonable for GP surgeries and $A \& E$ departments to distribute information sheets produced by the meningitis charities to worried parents.

It may be difficult to improve parental recognition of signs and symptoms of serious illness in their children. Aggressive public health campaigns, or information to parents such as the "baby check" have been shown to 
improve self referral rates. ${ }^{79} \mathrm{~A}$ public education programme was effective in reducing the time between illness onset and presentation during a recent epidemic of meningococcal disease in Norway, where specific information was disseminated about how to recognise the rash and symptoms of meningococcal disease.

Delayed recognition has been shown to be important in determining outcome of meningococcal disease and was confirmed in our study by the relation between duration of symptoms before treatment and mortality. ${ }^{9}$

Although the classical clinical features of meningitis (fever, vomiting, headache, neck stiffness, and photophobia), are recognised by most practising physicians, there is less awareness that the presenting features of meningococcal septicaemia are more commonly nonspecific (for example fever, rigors, headache, muscle pains, and confusion). These signs may wrongly be interpreted as an indication of influenza or other viral illness.

Since the original study by Steihm and Damrosch in 1966 that evaluated prognostic factors in meningococcal disease,${ }^{10}$ numerous studies have confirmed that those children who are most likely to die of meningococcal infection are those without meningitis, but with features of shock, disseminated intravascular coagulation, low white cell count in peripheral blood $\left(<10 \times 10^{9} / 1\right)$, low erythrocyte sedimentation rate $(<10 \mathrm{~mm} /$ hour $)$, low $C$ reactive protein $(<50 \mathrm{mg} / \mathrm{l})$, and a rapidly progressive purpuric rash. ${ }^{11-14}$ In this group of children, non-specific signs and symptoms will rapidly progress to organ failure and shock, which will be fatal in $20 \%-50 \%$ of cases, without features of meningitis ever being present. It may only be possible to diagnose these children as having meningococcal infection once the petechial rash becomes evident, or when features of inadequate end organ perfusion appear. Health professionals are therefore faced with the difficult task of picking out the "needle in a haystack": the one child with meningococcal infection from the many with trivial viral illness. Their task may be made easier by ensuring that they undress the child in a good light to try to identify the presence of a rash; by thorough examination, looking particularly for poor peripheral perfusion and tachycardia out of proportion to the degree of fever; noting the presence of persistent symptoms such as severe myalgia, often causing immobility; and severe abdominal pain. In the absence of these symptoms, clear instructions to the parents as stated above, or a planned review of the child a few hours later may act to reassure parents and doctors.

In assessing initial recognition of meningococcal disease by GPs and casualty officers, we used the same criteria which were defined as those which should have been recognised by parents as indicating serious illness in their child. Delayed recognition of meningococcal disease by GPs occurred in half of the children consulting their GP, despite the presence of a petechial rash and features of serious illness. The main problems in GP recognition that emerged from our study were: firstly, GPs may have failed to undress the child or examine a rash despite parental concern that a rash was present, and secondly, GPs may not have recognised features of serious illness, and therefore falsely reassured worried parents, thus making repeated consultation more difficult.

There will inevitably be debate as to the reliability of the signs of serious illness in children that we have used in this study. There may also be concerns that the parent's description of events, often recorded several hours after hospital admission, will be distorted. However even if methodological problems have resulted in some distortion of the events, there were many instances where reassurance was given by GPs or A\&E departments, despite a petechial rash or features of serious illness being present.

Although not all children had a positive microbiological diagnosis of meningococcal disease, the presence of a petechial rash and features of septicaemia or shock as defined are very specific for meningococcal disease in the UK. This, together with the absence of any alternative diagnosis and the knowledge that even without prior antimicrobial treatment only approximately $50 \%$ of individuals with meningococcal infection have positive microbiology allowed relative certainty of the diagnosis. ${ }^{15}$

Initial hospital management of children with meningococcal septicaemia was suboptimal in $70 \%$ of our cases. Half of the children with septicaemia and shock were not recognised as being shocked and therefore appropriate treatment was delayed. Even if shock was recognised, resuscitation was often inadequate. Lumbar puncture in the presence of cardiovascular instability or raised ICP is contraindicated. ${ }^{5}$ Knowledge of CSF changes in a child with the clinical features of meningococcal disease does not contribute to patient management and may be hazardous. This procedure was performed in 10 shocked children and one with raised ICP, nine of whom suffered significant clinical deterioration.

Thirteen children $(24 \%)$ died from meningococcal disease in this study. This is a higher mortality rate overall than that which is usually quoted. ${ }^{2}$ However, children referred to a tertiary centre are necessarily a more severely ill group. Forty two of 54 children had a diagnosis of meningococcal septicaemia. While this group of children was more severely ill, this does not bias the results of our study. If these children had been treated more appropriately, they may not have required referral to the PICU.

Our finding that only $29 \%$ of children with meningococcal septicaemia received optimal initial treatment suggests a need for improved training in the recognition and management of seriously ill children. Recent initiatives such as the widespread introduction of advanced life support courses (for example advanced paediatric life support and paediatric advanced life support) for junior paediatricians should go some way to addressing some of the issues of suboptimal initial hospital management. In 
addition, the use of treatment algorithms and clinical guidelines for hospital treatment may aid in the earlier recognition and institution of appropriate treatment for the seriously ill child. ${ }^{6}{ }^{16}$

\section{Conclusion}

This study has shown that delayed or suboptimal management occurs commonly in children with meningococcal disease. Failings occur in the delivery of all aspects of health care, from parental recognition, GP consultation, and hospital management. It cannot be concluded from this study whether outcome would have been improved if management had been optimal in every case. However, there are few diseases that progress as rapidly as meningococcal septicaemia, or in which relatively simple therapeutic interventions to correct the disordered physiology that is present are as critical to outcome. The impact on mortality of measures to improve public awareness of the disease and to improve management in the community and in hospital should be evaluated.

This work was supported by the Meningitis Research Foundation.

1 Jones D. Epidemiology of meningococcal disease in Europe and the USA. In: Cartwright $\mathrm{K}$, ed. Meningococcal disease. Chichester: John Wiley, 1995: 147-57.
2 Havens PL, Garland JS, Brook MM, et al. Trends in mortality in children hospitalised with meningococcal infections, 1957 to 1987. Pediatr Infect Dis J $1989 ; 8: 8-11$.

3 Bone RC. Let's agree on terminology: definitions of sepsis. Crit Care Med 1991;19:973-6.

4 Department of Health and Social Security. Meningococcal infection: meningitis and septicaemia. London: HMSO, 1988. (CMfection: mening (88) 2.)

5 Dean JM, Rogers MC, Traytsman RJ. Pathophysiology and clinical management of the intracranial vault. In: Roger MC, ed. Textbook of pediatric intensive care. Baltimore: Williams and Wilkins, 1992: 639-66.

6 Nadel S, Levin M, Habibi P. Treatment of meningococcal disease in childhood. In: Cartwright $\mathrm{K}$, ed. Meningococcal disease. Chichester: John Wiley, 1995: 207-43.

7 Morley CJ, Thonton AJ, Cole TJ, et al. Interpreting the symptoms and signs of illness in infants. In: David TJ, ed. symptoms and signs of illness in infants. In: David TJ, ed. Recent advances in paediatrics.
Livingstone, 1991: 137-55.

8 Steven N, Wood $M$. The clinical spectrum of meningococcal disease. In: Cartwright $\mathrm{K}$, ed. Meningococcal disease. Chichester: John Wiley, 1995: 177-205.

9 Gedde-Dahl TW, Hoiby EA, Brandtzaeg P, et al. Some arguments on early hospital admission and treatment of suspected meningococcal disease cases. National Institute of Public Health Annals 1990;13:45-60.

10 Steihm RS, Damrosch DS. Factors in the prognosis of meningococcal infection. J Pediatr 1966;68:457-67.

11 Lewis LS. Prognostic factors in acute meningococcaemia. Arch Dis Child 1979;54:44-8.

12 Leclerc F, Beuscart R, Guillois B, et al. Prognostic factors of severe infectious purpura in children. Intensive Care Med 1985;11:140-3.

13 Sinclair JF, Skeoch $\mathrm{CH}$, Hallworth D. Prognosis of meningococcal septicaemia. Lancet $1987 ;$ ii 38 .

14 Gedde-Dahl TW, Bjark P, Høiby EA, et al. Severity of meningococcal disease: assessment by factors and scores and implications for patient management. Rev Infect Dis 1990;12:973-91.

15 Cartwright KV, Jones DM. Investigation of meningococcal disease. J Clin Pathol 1989;42:634-9.

16 Hodgetts T, Brett A, Castle N. The early management of meningococcal disease. J Accid Emerg Med 1998;15:72-6. 